\title{
Electron Stripping Cross Sections for Fast, Low Charge State Uranium Ions
}

\author{
R. E. Olson ${ }^{1}$, R. L. Watson ${ }^{2}$, V. Horvat ${ }^{2}$, K. E. Zaharakis ${ }^{2}$, R. D. DuBois ${ }^{1}$ and
}

Th. Stöhlker ${ }^{3}$

${ }^{1}$ Department of Physics, University of Missouri-Rolla, Rolla, MO 65401

${ }^{2}$ Cyclotron Institute and Department of Chemistry, Texas A\&M University, College

Station, TX 77843

${ }^{3}$ Atomphysik, GSI-Darmstadt, D-64291 Darmstadt, Germany

\begin{abstract}
Cross sections for projectile electron loss for $\mathrm{U}^{10+}$ and $\mathrm{U}^{28+}$ colliding with $\mathrm{H}_{2}, \mathrm{~N}_{2}$ and $\mathrm{Ar}$ were calculated using the n-body classical trajectory Monte Carlo method. The calculations include electrons on both nuclear centers and all electron-electron and electron-nuclear interactions between centers. Multiple ionization is inherently included in these many electron calculations. Overall, except for the $\mathrm{H}_{2}$ target where the projectile stripping is overestimated by a factor of two, the calculated cross sections are in reasonable accord with available data and the recent beam lifetime measurements from GSI-Darmstadt. For energies less than $100 \mathrm{MeV} / \mathrm{u}$, the $\mathrm{N}_{2}$ and Ar cross sections do not scale as $\mathrm{E}^{-1.0}$ as predicted by one-electron theories.
\end{abstract}

PACS: $\quad$ 34.50Fa, 34.50.Bw, 29.27.Eg

Keywords: electron loss, stripping, fusion, beam transport 


\section{Introduction}

For the heavy ion driven, hot dense plasma experiments planned in both the U.S. and Germany, a partially-stripped heavy ion is accelerated to $\mathrm{MeV} / \mathrm{u}$ energies and focused onto a target. A low charge state ion is required to reduce space charge expansion of the beam in the focusing region. However, at MeV/u energies, low charge state projectiles are subject to considerable stripping of their electrons. This projectile electron loss results in degraded focusing, and in the accelerator structure itself, loss of the beam to the walls. For the latter, these charge-changed fast ions strike the accelerator container walls and give rise to sputtering and activation of the surfaces.

The German program has considered low charge state uranium ions such as $\mathrm{U}^{10^{+}}$, but now most interest is centered on $\mathrm{U}^{28+}$ for energies up to about $200 \mathrm{MeV} / \mathrm{u}$. A new SIS-100/300 synchrotron facility is being built at GSI-Darmstadt. Projectile stripping cross sections are needed in order to assess the vacuum requirements of the ring. Unfortunately, little to no data exist for projectile ions at the charges and energies of interest. This is because the ion charge states are far below their equilibrium values where experiments can be conveniently performed. Moreover, low charge to mass ratio and high energies are not compatible with existing accelerators. Thus, it is necessary to rely on theoretical calculations for predictions. For these uranium ion systems, only the one-electron Born calculations of Shevelko et al [1,2] are available.

To test the many-electron computations presented here, experiments were conducted at the Texas A\&M Cyclotron Institute. The experiment [3] provided projectile charge changing (stripping plus electron capture) total and partial cross sections at 
energies of 3.5 and $6.5 \mathrm{MeV} / \mathrm{u}$. These data were supplemented with cross sections measured several decades ago at $1.4 \mathrm{MeV} / \mathrm{u}$ by Erb [4] and Franzke [5] at GSIDarmstadt. Except for the hydrogen target, multiple electron removal was found to be very important and made up approximately 50\% of the total projectile electron loss cross section.

\section{Method}

Our calculations employ the n-body classical trajectory Monte Carlo method nCTMC [6,7]. All relevant ionizing interactions, i.e. those between particles on the two centers, are considered in an exact way. The Hamiltonian is given by

$$
\mathrm{H}=\left(\mathrm{H}_{\mathrm{o}}+\mathrm{V}_{\mathrm{NP}-\mathrm{eP}}+\mathrm{V}_{\mathrm{NT}-\mathrm{eT}}\right)+\mathrm{V}_{\mathrm{NP}-\mathrm{NT}}+\mathrm{V}_{\mathrm{NT}-\mathrm{eP}}+\mathrm{V}_{\mathrm{NP}-\mathrm{eT}}+\mathrm{V}_{\mathrm{eP}-\mathrm{eT}}
$$

where $\mathrm{H}_{\mathrm{o}}$ is the kinetic energy and the potential interaction subscripts are denoted by NP (projectile nucleus), NT (target nucleus), eP (projectile electron), and eT (target electron). Ionization on one or both centers is produced by the combined and competing (N-e) and (e-e) interactions.

The n-body CTMC calculations are computer intensive. For the $\mathrm{U}^{28+}$ ion, it was necessary to utilize 36 outer shell electrons $\left(4 s^{2} 4 p^{6} 4 d^{10} 4 f^{14} 5 s^{2} 5 p^{2}\right)$ in order to obtain convergence on the total cross section. All electrons on the targets were incorporated in the calculations except in the case of Ar where it was deemed unnecessary to include the K-shell electrons. Thus, counting the parent nuclei, the number of bodies used in the calculations amounted to 39, 45, and 54 for the H, N and Ar targets, respectively.

For $\mathrm{U}^{10+}$, convergence tests indicated that the inclusion of the $\left(5 s^{2} 5 p^{6} 5 d^{10} 6 s^{2} 6 p^{6}\right)$ electrons was sufficient to obtain accurate cross sections. The number of bodies included in these calculations were 28, 34, and 43 for the $\mathrm{H}, \mathrm{N}$ and Ar targets, respectively. 
All single and multiple ionization processes are inherently included within the nCTMC method along with conservation of flux, both of which are ignored within single electron models. Further, since the nCTMC method follows the energy deposition to the heavy particles and uses this information, along with the experimental sequential binding energies to determine the ionization levels, at least to first order, multiple excitation and subsequent Auger ionization are approximated by the calculations.

\section{Projectile Stripping Cross Sections}

\section{A. $\mathbf{U}^{10+}$}

The calculated cross sections are displayed in fig. 1 along with available experimental data. All 1.4 MeV/u data were measured at GSI. For the $\mathrm{N}_{2}$ system, the nCTMC results are in agreement with the work of Franzke [5], but lie a factor of two above those of DuBois et al. For $\mathrm{H}_{2}$, the calculations overestimate the data by a factor of two.

B. $\mathbf{U}^{28+}$

The nCTMC cross sections for $\mathrm{U}^{28+}$ are presented in figs. 2-4. As in the case of $\mathrm{U}^{10+}$, the $\mathrm{H}_{2}$ data are overestimated by a factor of two. For the heavier targets the calculations are within $30 \%$ of all data. Note also the difference in the energy dependence of the cross sections predicted by the Born and nCTMC methods for the $\mathrm{N}_{2}$ and Ar cases. This difference has a significant affect on the beam lifetime predicted for high energies.

\section{Ion Beam Lifetimes}

The calculated $\mathrm{U}^{28+}$ stripping cross sections presented above were used to estimate the beam lifetime for the SIS ring at GSI-Darmstadt and compared with recent 
measurements [8]. The vacuum constituents were measured to be $\mathrm{H}_{2}$ at $65 \%, \mathrm{H}_{2} \mathrm{O}$ at $17 \%, \mathrm{CO} / \mathrm{N}_{2}$ at $8 \%, \mathrm{Cl}$ at $4 \%, \mathrm{Ar}$ at $4 \%$, and $\mathrm{CO}_{2}$ at $1 \%$. The vacuum pressure in the accelerator was found to be $7.7 \times 10^{-11}$ mbar. The target Z-scaling published by Watson et al. [3] was used to predict the cross sections for those vacuum constituents not given by the $\mathrm{H}_{2}, \mathrm{~N}_{2}$ and Ar systems studied above. As a representative contribution to the beam lifetime, when the vacuum constituent fractions given were weighted by the theoretical cross sections at $20 \mathrm{MeV} / \mathrm{u}$, the H-system contributed 13\%, N 62\%, and Ar 24\%. Thus, although the nCTMC cross sections overestimate the $\mathrm{H}_{2}$ data by a factor of two, this inaccuracy amounted to only a 7\% decrease in the overall calculated beam lifetime.

The calculated and observed beam lifetimes are shown in Fig. 5. The nCTMC results overestimate the measured beam lifetime by about $25 \%$, but display a similar energy dependence. This is in contrast to Born calculations, whose $\mathrm{E}^{-1}$ high energy cross section dependence leads to an over optimistic estimate of the lifetime and an energy dependence that increases as $\mathrm{E}^{+0.5}$.

The beam lifetime for $\mathrm{U}^{10+}$ can be easily estimated from the cross sections given above. We conclude that under the same vacuum conditions as measured for the $\mathrm{U}^{28^{+}}$ system, the beam lifetime for $\mathrm{U}^{10+}$ will be approximately six times lower than that of $\mathrm{U}^{28+}$, and will have a similar energy dependence.

\section{Acknowledgements}

Work supported by the DOE Office of Fusion Energy Sciences and the Robert A. Welch Foundation. 


\section{References}

[1] V. P. Shevelko, D. Böhne and Th. Stöhlker, Nucl. Instr. Meth. A 415 (1998) 609.

[2] V. P. Shevelko, I. Yu Tolstikhina and Th. Stöhlker, Nucl. Instr. Meth. B 184 (2001) 295.

[3] R. L. Watson, Y. Peng, V. Horvat, G. J. Kim and R. E. Olson, Phys. Rev. A 67 (2003) 022706.

[4] W. Erb, GSI Report P-7-78, October (1978).

[5] B. Franzke, IEEE NS-28 (1981) 2116.

[6] R. E. Olson, J. Ullrich and H. Schmidt-Böcking, Phys. Rev. A 39 (1989) 5572.

[7] R. E. Olson, Atomic, Molecular, and Optical Physics Handbook, ed. by G. W. F. Drake, AIP, (1996) Chap. 56.

[8] A. Krämer, O. Boine-Frankenheim, E. Mustafin, H. Reich-Sprenger and P. Spiller, Proc. EPAC2002, (2002) Paris.

web address: http://accelconf.web.cern.ch/AccelConf/e02/PAPERS/WEBLE116.pdf 
Figures

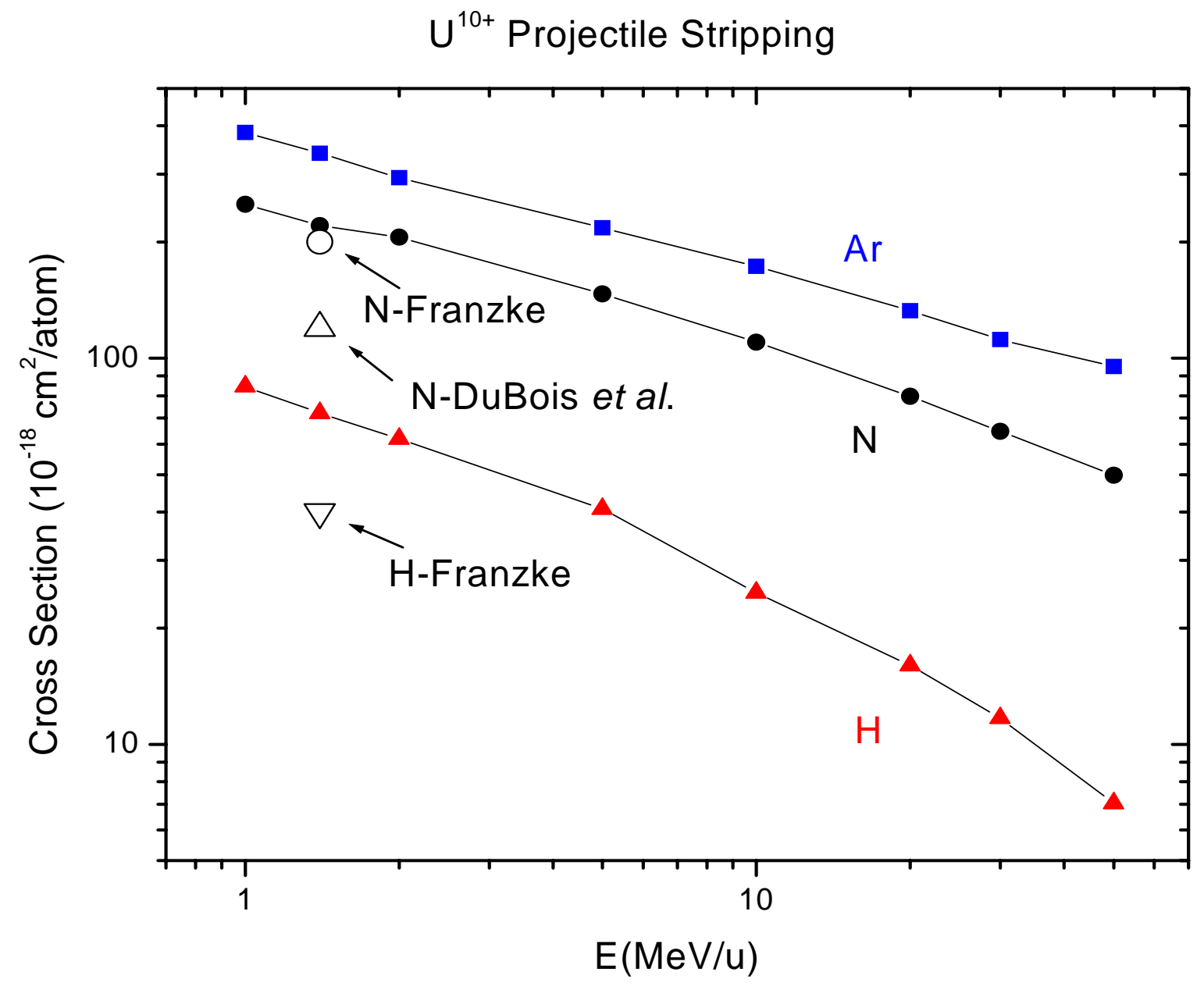

Figure 1: Total $\mathrm{U}^{10^{+}}+\mathrm{H}_{2}, \mathrm{~N}_{2}$ and Ar projectile stripping cross sections as calculated by the nCTMC method. Experimental data at 1.4 MeV/u are from Franzke [5] and DuBois et al. 


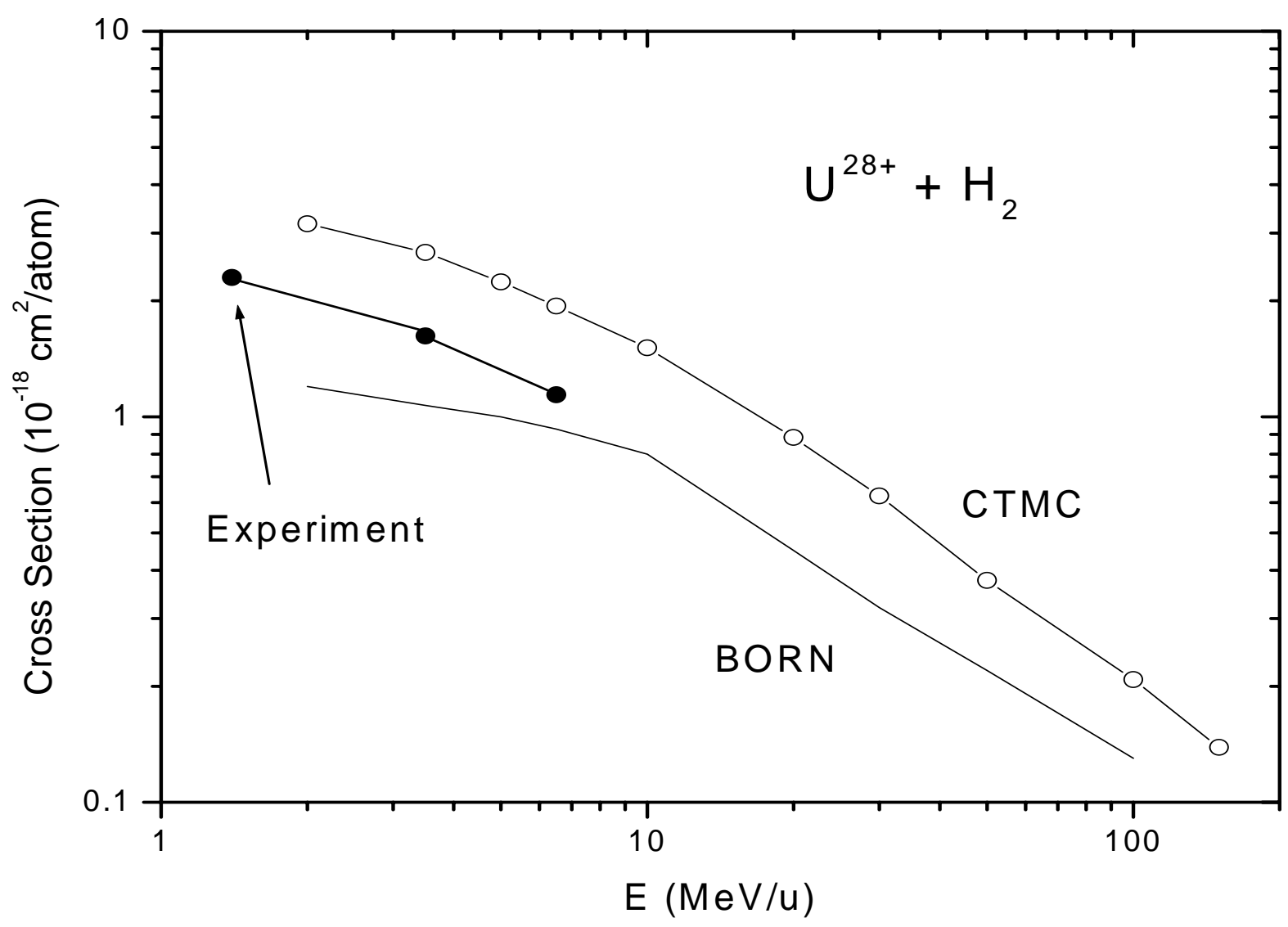

Figure 2: $\mathrm{U}^{28+}$ stripping collisions for impact on $\mathrm{H}_{2}$. The experimental data at $1.4 \mathrm{MeV} / \mathrm{u}$ are from Franzke [5] and the 3.5 and $6.5 \mathrm{MeV} / \mathrm{u}$ results are from Texas A\&M. The Born approximation calculations are from Shevelko et al. [1,2]. 


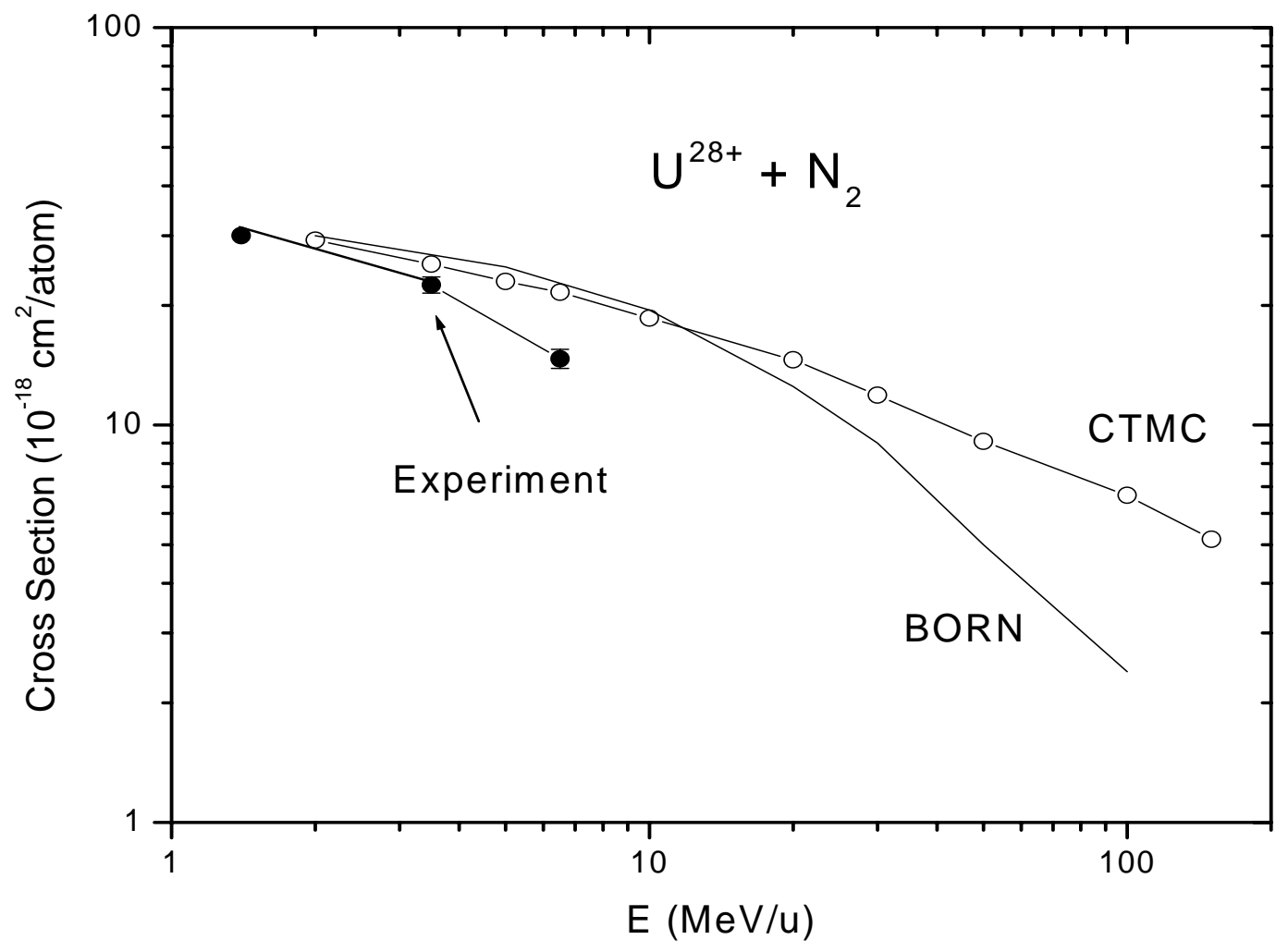

Figure 3: $\mathrm{U}^{28+}$ stripping collisions for impact on $\mathrm{N}_{2}$. The experimental data at $1.4 \mathrm{MeV} / \mathrm{u}$ are from Franzke [5] and the 3.5 and $6.5 \mathrm{MeV} / \mathrm{u}$ results are from Texas A\&M. The Born approximation calculations are from Shevelko et al. [1,2]. 


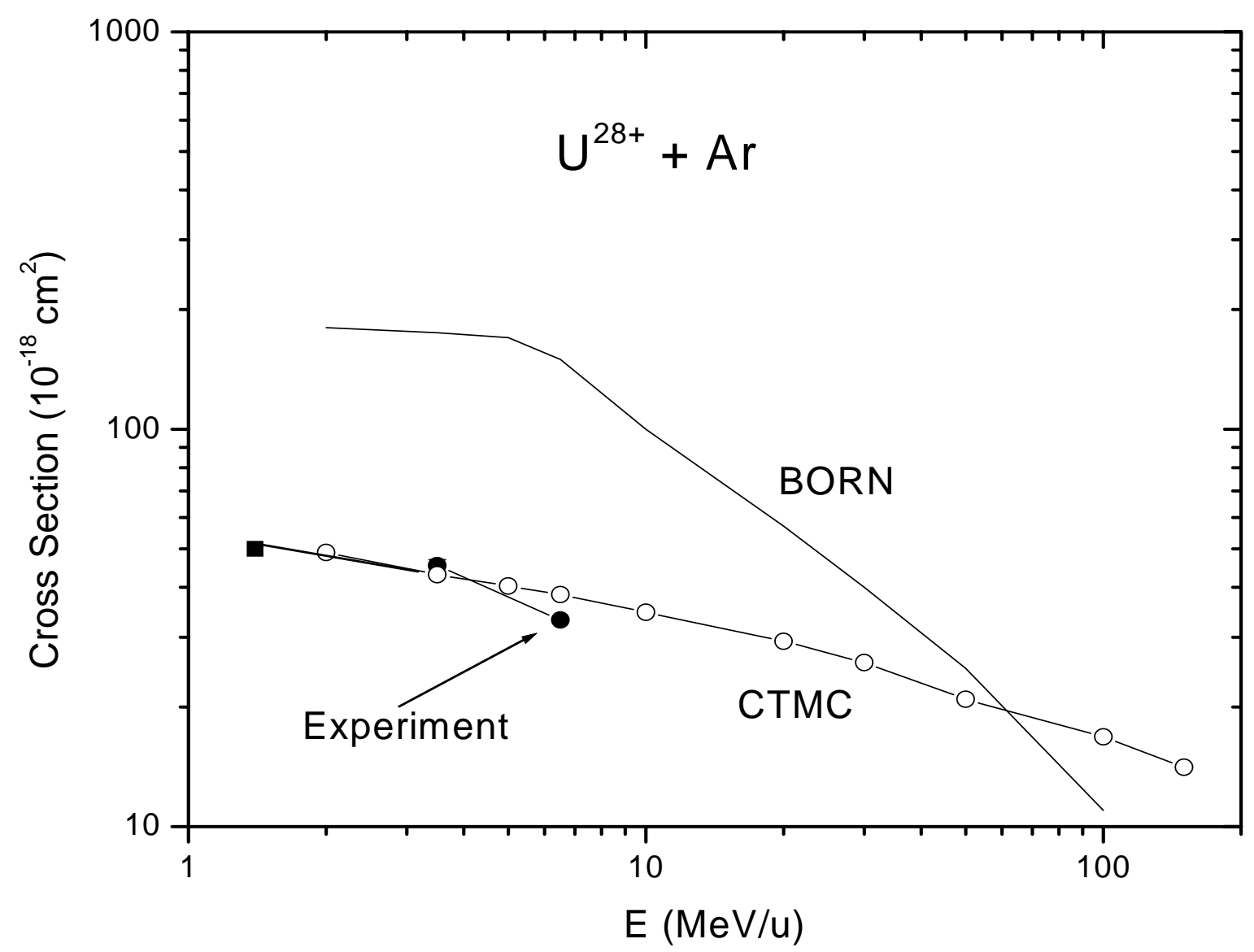

Figure 4: $\mathrm{U}^{28+}$ stripping collisions for impact on Ar. The experimental data at $1.4 \mathrm{MeV} / \mathrm{u}$ are from Erb [4] and the 3.5 and $6.5 \mathrm{MeV} / \mathrm{u}$ results are from Texas A\&M. The Born approximation calculations are from Shevelko et al. [1,2]. 


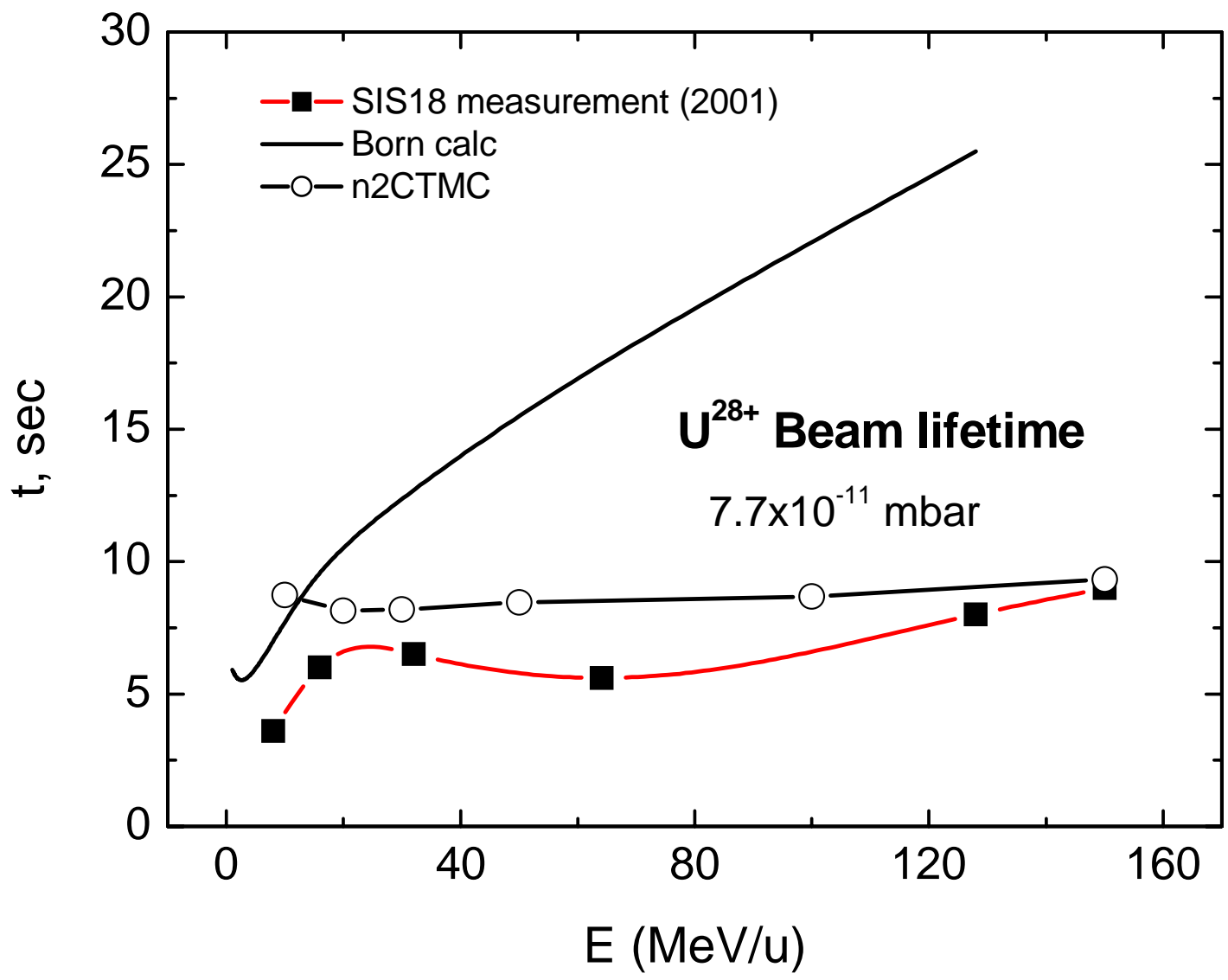

Figure 5: $\mathrm{U}^{28+}$ beam lifetime as a function of energy for a pressure of $7.7 \times 10^{-11} \mathrm{mbar}$ measured at the GSI SIS storage ring (Krämer et al. Ref. 8). The open circles are the nCTMC results from the cross sections presented above, while the line increasing as approximately $\mathrm{E}^{+0.5}$ are from the Born calculations of Shevelko [2]. 